\title{
Comparison of the retention strengths of three complete denture adhesives: An in vivo study
}

\author{
Jose F. Mañes ${ }^{1}$, Eduardo J. Selva ${ }^{2}$, Alfonso De-Barutell ${ }^{2}$, Kheira Bouazza ${ }^{3}$

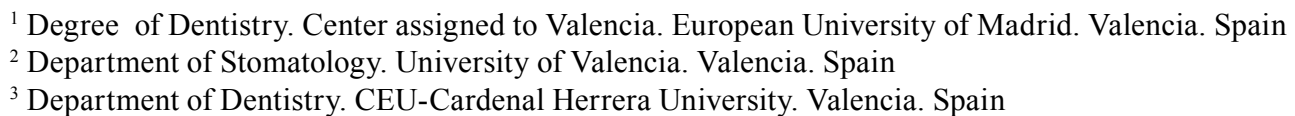

Correspondence:

Department of Stomatology

University of Valencia

Valencia. Spain

C/ Gascó Oliag 1

46010 - Valencia (Spain)

Eduardo.J.Selva@uv.es

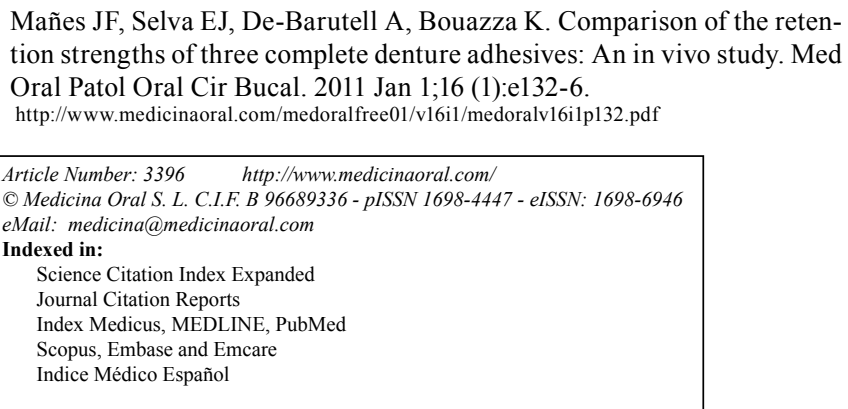

\begin{abstract}
Statement of problem: One of the main problems posed by complete dentures is retention and stability. In order to solve this problem, dentists and the dental industry for a long time have attempted to improve denture adhesion by developing a range of "glues" of highly varied composition and efficacy. Purpouse: The present in vivo clinical study evaluates whether the adhesives used to improve complete denture retention are truly effective and able to increase denture adhesion to the mucosa covering the edentulous alveolar ridge of the mandibular dentures. Material and Methods: An in vivo clinical study is made of 30 patients with complete mandibular dentures to evaluate the retention afforded by three commercial complete denture adhesives (Benfix ${ }^{\circledR}$, Fittydent ${ }^{\circledR}$ and Supercorega ${ }^{\circledR}$. A spring scale was used to measure retention strength (in grams). The purpose was to determine whether the use of complete denture adhesives is effective, and to establish which commercial brands offer the highest retention strengths. Results and Conclusion:The results obtained indicate that retention is enhanced by the use of such adhesives, and that Fittydent ${ }^{\circledR}$ offers the best retention performance, followed by Benfix ${ }^{\circledR}$ and Supercorega ${ }^{\circledR}$. Clinical implications: the study of denture adhesives and his efficiency are necessary to improve the edentulous patient satisfaction. More in vivo investigations are necessary in dental literature.
\end{abstract}

Key words: Retention, complete dentures, adhesives of dentures.

\section{Introduction}

One of the main problems posed by complete dentures is retention and stability of the mandibular dentures. In order to solve this problem, dentists and the dental industry for a long time have attempted to improve denture adhesion by developing a range of "glues" of highly varied composition and efficacy.

The use of dental adhesives began in the XVIII century
(1). These products were prepared by pharmacists who mixed plant gums to produce a material that could absorb the humidity of saliva and swell to form a mucilaginous layer adhering to the oral mucosa and dentures. Dental adhesives are used in prosthodontics to provide a binding layer on the surface of removable complete dentures (1), thus allowing the latter to adhere to the supporting tissues of the edentulous patient. Basically, 
denture adhesives act by increasing the viscosity of saliva and of the interface between the dentures and mucosa, thus facilitating peripheral sealing (2).

Dental adhesives are composed of three large groups of materials. A first group comprises the actual adhesives, including a broad range of classical products such as plant gums (karaya, tragacanth, acacia), and more recent components based on natural (methylcellulose, hydroxymethyl cellulose, carboxymethyl cellulose) and synthetic polymers (polyethylene oxide, arcylamides, polyvinyl acetate) (3). A second group of materials comprises antimicrobial agents such as sodium borate, sodium tetraborate, hexachlorophene or propylhydroxybenzoate and ethanol (4). These substances act as preservatives and are moderately active against Candida albicans. The third group of materials in turn consists of binding agents, humectants (sodium laurylsulfate), flavoring agents (mint or natural oils of salvia, olive, chamomile, etc.) and plastifiers.

The present in vivo clinical study evaluates whether the adhesives used to improve complete denture retention are truly effective and able to increase denture adhesion to the mucosa covering the edentulous alveolar ridge. Retention is quantified, and a statistical analysis is made to determine which of three commercial complete denture adhesives offer the best retention performance.

\section{Material and Methods}

Three leading commercial complete denture adhesives were selected, supplied in the form of creams and with a similar formulation in all three cases: Benfix ${ }^{\circledR}$ (Vitafarma SL, Hernani, Spain), Fittydent ${ }^{\circledR}$ (Fittydent Int. GMBH, Pinkafeld, Austria) and Supercorega ${ }^{\circledR}$ (Stafford-Miller, Dungarvan Co. Waterford, Ireland). Quantification of retention without adhesive was also carried out as control.

The study was carried out in 30 patients with removable complete dentures, independently of how long the dentures had been worn, or of their quality and retention performance. After the obtainment of informed consent in all cases, the patients were instructed to maintain maximum, non-forced intercuspidation during 5 minutes. After this time, and with the mouth open and the lower lip relaxed in order to avoid losing peripheral sealing, the tip of a spring scale (Carpo, France) was placed at the margin of the dentures, in the recess of the lower lingual frenulum (Fig. 1). Traction was then applied until the dentures detached - the maximum retention force being registered by the spring scale (Fig. 2).

After recording retention of the mandibular dentures, one of the study adhesives was applied. The same amount of adhesive was used in all tests, distributing the material in three equivalent portions in the anterior and lateral zones, in compliance with the instructions of the manufacturers (Fig. 3).

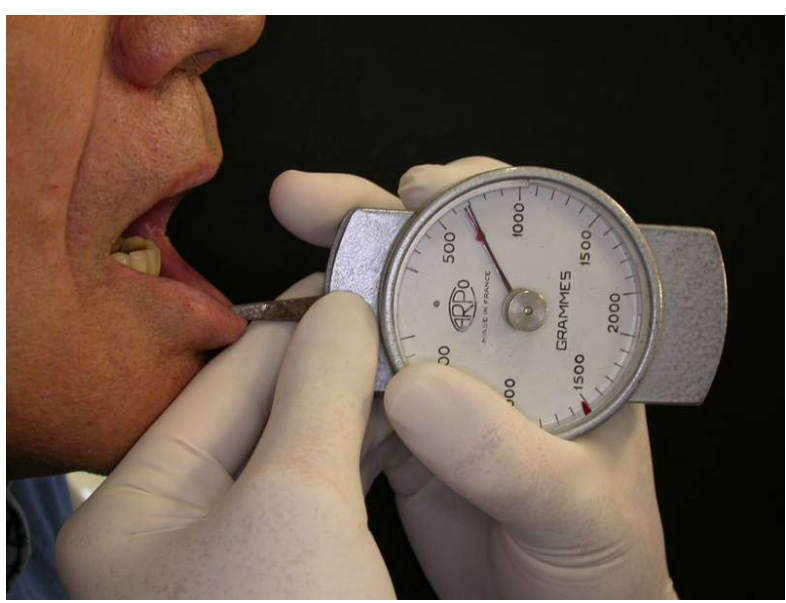

Fig. 1. The tip of a spring scale (Carpo, France) was placed at the margin of the dentures.

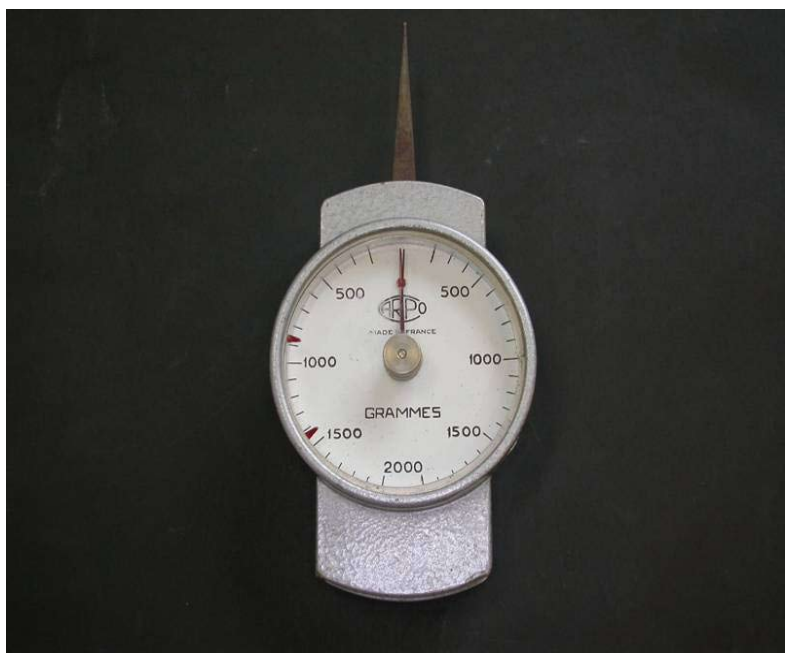

Fig. 2. The maximum retention force being registered by the spring scale.

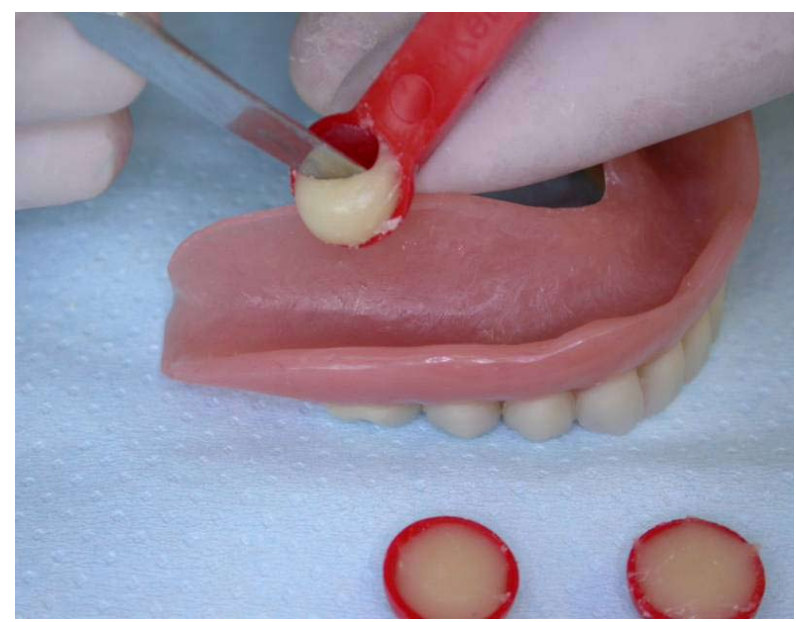

Fig. 3. The same amount of adhesive was used in all tests. 
The dentures were then placed in the mouth, and the patients were again instructed to maintain maximum, non-forced intercuspidation during 5 minutes. After this time retention force (in grams) was again recorded as described above.

This procedure was repeated three times for each product, without adding further amounts of adhesive, and waiting one minute in occlusion after each measurement.

After completing the study with each adhesive, the dentures were thoroughly cleaned using the means recommended by the manufacturers, in order to eliminate any possible summing of effects among materials. The same procedure as described above was then repeated with the next product. The order of application of the adhesives was randomized, and all measurements were made by the same investigator.

Thus, each patient was subjected to three measurements of retention strength without adhesive (control values), together with three measurements involving each of the three commercial adhesives.

\section{Results}

In this repeated measures study, and with the purpose of eliminating possible differences in retention capacity attributable to the sequence in which the adhesives were applied, the latter were previously selected on a random basis.

Calculations were made of the arithmetic means of the three retention force measurements (in grams) corresponding to each adhesive (Benfix ${ }^{\circledR}$, Fittydent ${ }^{\circledR}$ and Supercorega $^{\circledR}$ in random sequence) and without adhesive (control values).

The adhesive performances of the different products were compared, and the effects of the sequence of application were evaluated.

Statistical significance was accepted for $p<0.05$. (Table 1) shows the mean retention forces (in grams) for all four series of measurements $\left(\right.$ Benfix $^{\circledR}$, Fittydent $\AA$, Supercorega ${ }^{\circledR}$, and control). Retention was markedly low in the absence of adhesive (202 $\pm 273 \mathrm{~g}$ ), while the highest values corresponded to Fittydent ${ }^{\circledR}(1095 \pm 668 \mathrm{~g})$. However, the important dispersion of values observed with each adhesive (Table 2) recommended the use of the median as descriptive statistic. Thus, in the absence of adhesive (control), the median retention was $58 \mathrm{~g}$, while Fittydent ${ }^{\circledR}$ reached 875 g. Supercorega ${ }^{\circledR}$ and Benfix $^{\circledR}$ in turn yielded intermediate strengths.

The nonparametric Wilcoxon test for repeated measures was applied to determine whether the retentions obtained with each commercial adhesive were superior to those recorded in the absence of adhesive. The results showed that all the adhesives, regardless of the commercial brand involved, significantly improved retention versus the control values.

The existence of differences among the three commercial adhesives was evaluated by analysis of variance

Table 2. Dispersion plot.

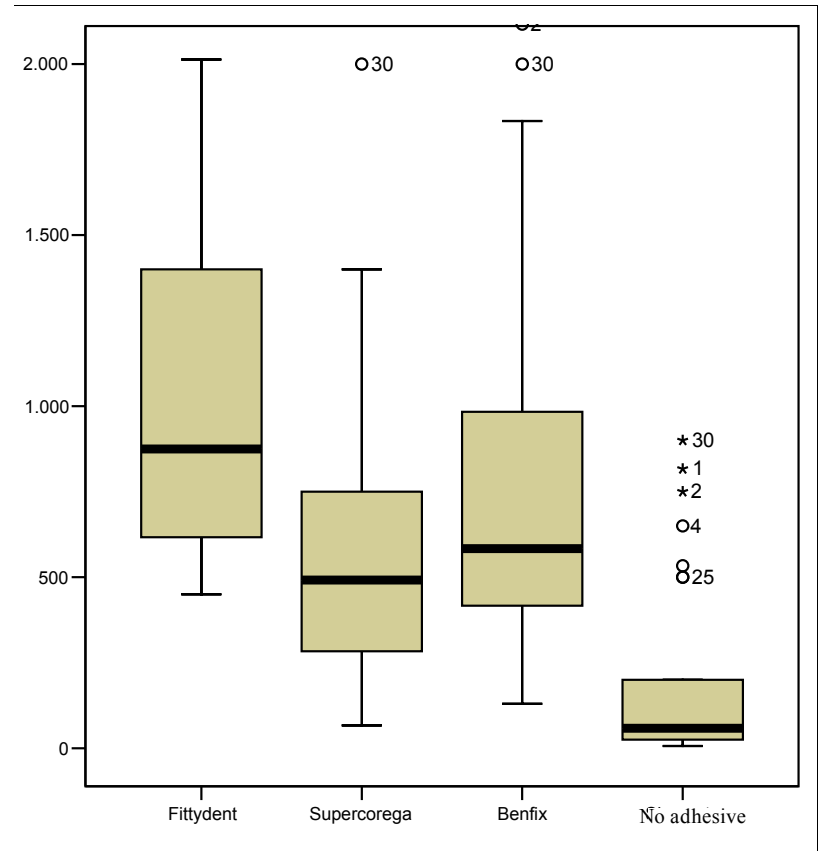

Table 1. Mean retention values (in grams).

\begin{tabular}{|l|c|c|c|c|c|c|}
\hline & Count & Mean & St. dev. & Median & Minimum & Maximum \\
\hline Fittydent $^{\circledR}$ & 30 & 1095.17 & 668.64 & 875.00 & 450.00 & 3466.67 \\
\hline Supercorega $^{\circledR}$ & 30 & 560.11 & 407.19 & 491.67 & 66.67 & 2000.00 \\
\hline Benfix $^{\circledR}$ & 30 & 846.56 & 818.21 & 583.33 & 130.00 & 4116.67 \\
\hline No adhesive & 30 & 202.06 & 273.15 & 58.33 & 6.67 & 900.00 \\
\hline
\end{tabular}


(ANOVA) for repeated measures (since the data showed a normal distribution), considering the brand and order of application. The mean retention values recorded for each commercial adhesive were seen to be independent of the order of adhesive application, i.e., no order or sequence effect was observed. On the other hand, the adhesive performances of the three commercial products were seen to differ significantly $(\mathrm{p}<0.001)$. Specifically, the best adhesive performance corresponded to Fittydent ${ }^{\circledR}$, followed by Benfix $x^{\circledR}$ and finally Supercorega ${ }^{\circledR}$.

\section{Discussion}

Dentists seem to be scantly inclined to recommend the use of complete denture adhesives. In effect, the use of such products appears to correspond more to patient desires for a solution to mobility and retention problems than to dental prescription. This may be due to a lack of confidence in the results obtained, the purported iatrogenic problems of such products (5), or concern that patients may replace adequate denture maintenance with different commercial adhesives (powders, creams, and particularly cushions).

Dentists are aware that the success of removable dentures is fundamentally dependent upon the achievement of adequately balanced occlusion affording stability, together with maximum extension of the bases to secure the key to retention, i.e., adequate peripheral sealing. In any case, many clinicians are uncertain about the true efficacy of such products, and are not sure about which adhesives offer the best retention performance. Many authors (6-8) consider these products to improve denture stability and retention, though almost all the research published in the literature consists of in vitro studies (9) and/or is based on the administration of patient satisfaction questionnaires $(10,11)$. Of the limited in vivo studies carried out to date, most involve the upper maxilla (12), and only a few involve the mandible (13). We therefore designed the present study to compare the efficacy of these products in the course of several hours of use.

Mandibular dentures were used because they normally pose the greatest retention problems. The patients were included independently of how long the dentures had been worn, or of their quality, since the study protocol contemplated repeated measurements in one same patient and involving the same dentures.

One of the problems associated with studies of this kind is the need for a system capable of registering retention, with simple handling characteristics and good patient tolerance (12-14). In this study we used a very simple spring scale that proved comfortable for the patients. Traction is applied to the anterior zone of the dentures, which may be interpreted as a source of error in terms of the quantification of retention. Nevertheless, this problem is not important provided peripheral sealing of the dentures is maintained, since the study involved measurements in the same patient, with the same dentures, and varying only the type of adhesive (or no adhesive) used. The measurements were obtained following 5 minutes of non-forced occlusion.

After applying traction to the point of dentures detachment, the latter were repositioned and occlusion was maintained for one minute. In all cases this gave rise to lower traction force values after the second and third detachments, possibly due to a lack of adequate adaptation of the adhesive material to the edentulous alveolar ridge.

In coincidence with the literature $(15,16)$ our results indicate that the adhesives effectively increase the retention of complete dentures.

Although the composition of the products used was very similar, significantly higher retention values were recorded for Fittydent ${ }^{\circledR}$ versus the other two adhesives - possibly because of its greater viscosity and low water solubility. Psillakis (17) conducted a mixed study involving the use of a gnathometer to measure the force needed to detach the dentures, and the administration of a subjective patient questionnaire to assess chewing, comfort and confidence. A $64 \%$ increase in dentures retention was observed when using dental adhesive, and $74 \%$ of the patients reported improved denture function with adhesive. In comparison, a few minutes after placing the adhesive, we recorded an over 10- fold increase in retention strength. However, the above study involved upper dentures, which tend to show better retention than mandibular dentures, and this may explain the differences in results. This retention strength can be expected to decrease over time as the saliva gradually dissolves the adhesive material (18).

\section{Conclusions}

The use of adhesive creams with removable complete mandibular dentures significantly increases their retention.

Of the three commercial adhesives considered in the present study, the best retention performance corresponded to Fittydent ${ }^{\circledR}$, followed by Benfix ${ }^{\circledR}$ and finally Supercorega ${ }^{\circledR}$.

\section{References References with links to Crossref-DOI}

1. McCabe JF, Carrick TE, Kamohara H. Adhesive bond strength and compliance for denture soft lining materials. Biomaterials. 2002;23:1347-52.

2. Grasso JE. Denture adhesives: changing attitudes. J Am Dent Assoc. 1996;127:90-6.

3. Love WB, Biswas S. Denture adhesives--pH and buffering capacity. J Prosthet Dent. 1991;66:356-60.

4. Gates WD, Goldschmidt M, Kramer D. Microbial contamination in four commercially available denture adhesives. J Prosthet Dent. 1994;71:154-8.

5. Ekstrand K, Hensten-Pettersen A, Kullmann A. Denture adhesives: cytotoxicity, microbial contamination, and formaldehyde content. J Prosthet Dent. 1993;69:314-7. 
6. Kapur KK. A clinical evaluation of denture adhesives. J Prosthet Dent. 1967;18:550-8.

7. Berg E. A clinical comparison of four denture adhesives. Int $\mathrm{J}$ Prosthodont. 1991;4:449-56.

8. Chew CL, Boone ME, Swartz ML, Phillips RW. Denture adhesives: their effects on denture retention and stability. J Dent. 1985;13:152-9.

9. Koppang R, Berg E, Dahm S, Real C, Fløystrand F. A method for testing denture adhesives. J Prosthet Dent. 1995;73:486-91.

10. Uysal H, Altay OT, Alparslan N, Bilge A. Comparison of four different denture cushion adhesives--a subjective study. J Oral Rehabil. 1998;25:209-13.

11. Kelsey CC, Lang BR, Wang RF. Examining patients' responses about the effectiveness of five denture adhesive pastes. J Am Dent Assoc. 1997;128:1532-8.

12. Kikuchi M, Ghani F, Watanabe M. Method for enhancing retention in complete denture bases. J Prosthet Dent. 1999;81:399-403.

13. Mirza FD, Dikshit JV, Murdia NS. Comparative study of effectiveness of denture adhesives at different time intervals. J Indian Dent Assoc. 1984;56:335-7.

14. Ow RK, Bearn EM. A method of studying the effect of adhesives on denture retention. J Prosthet Dent. 1983;50:332-7.

15. Coates AJ. Denture adhesives: a review. Aust Prosthodont J. 1995;9:27-31

16. Olshan AM, Ross NM, Mankodi S, Melita S. A modified Kapur scale for evaluating denture retention and stability: methodology study. Am J Dent. 1992;5:88-90.

17. Psillakis JJ, Wright RF, Grbic JT, Lamster IB. In practice evaluation of a denture adhesive using a gnathometer. J Prosthodont. 2004;13:244-50.

18. Chew CL. Retention of denture adhesives--an in vitro study. J Oral Rehabil. 1990;17:425-34. 